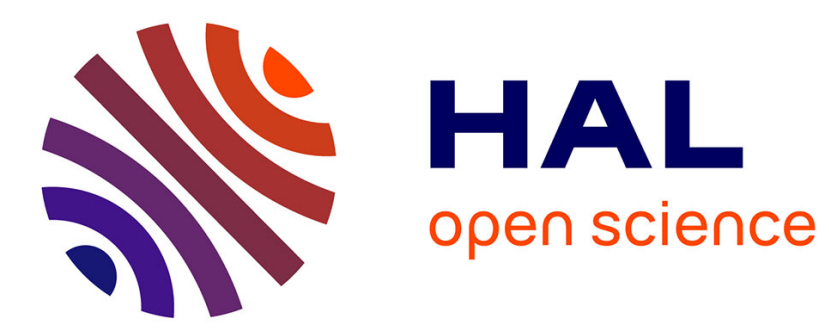

\title{
RÔLE DU CONTACT PHYSIQUE DANS LA MANIFESTATION DES RELATIONS HIÉRARCHIQUES CHEZ LES BOVINS. CONSÉQUENCES PRATIQUES
}

\author{
Marie-France Bouissou, Colette Lavenet, P. Orgeur
}

\section{To cite this version:}

Marie-France Bouissou, Colette Lavenet, P. Orgeur. RÔLE DU CONTACT PHYSIQUE DANS LA MANIFESTATION DES RELATIONS HIÉRARCHIQUES CHEZ LES BOVINS. CONSÉQUENCES PRATIQUES. Annales de zootechnie, 1970, 19 (3), pp.279-285. hal-00887009

\section{HAL Id: hal-00887009 https://hal.science/hal-00887009}

Submitted on 1 Jan 1970

HAL is a multi-disciplinary open access archive for the deposit and dissemination of scientific research documents, whether they are published or not. The documents may come from teaching and research institutions in France or abroad, or from public or private research centers.
L'archive ouverte pluridisciplinaire $\mathbf{H A L}$, est destinée au dépôt et à la diffusion de documents scientifiques de niveau recherche, publiés ou non, émanant des établissements d'enseignement et de recherche français ou étrangers, des laboratoires publics ou privés. 
Ann. Zootech., I970, 19 (3), 279-285.

\title{
RÔLE DU CONTACT PHYSIQUE DANS LA MANIFESTATION DES RELATIONS HIÉRARCHIQUES CHEZ LES BOVINS. CONSÉQUENCES PRATIQUES
}

\author{
Marie-France BOUISSOU \\ avec la collaboration technique de Colette LAvenet et P. Orgeur \\ Station de Physiologie de la Reproduction, \\ Centre de Recherches de Tours, 37 - Nouzilly \\ Institut national de la Recherche agronomique
}

\section{RÉSUMÉ}

L'étude des signaux de dominance-soumission chez les Bovins domestiques a mis en évidence le rôle important de la possibilité d'un contact physique entre les animaux.

L'absence de cette possibilité permet d'effacer les conséquences défavorables de l'existence de la hiérarchie sur le temps d'alimentation des animaux dominés lors d'une compétition avec un dominant.

Une étude expérimentale de cette "notion de protection " a permis de montrer que la protection au niveau de la tête en était l'élément essentiel.

\section{IN'TRODUCTION}

Les phénomènes de hiérarchie sociale sont largement répandus dans le monde animal parmi les espèces vivant naturellement ou étant artificiellement maintenues en groupe, et ont été également trouvés chez les Bovins domestiques (ScHEIN et Fohrman, I955 ; Guhl, et AtKeson, I959 ; Beil,HARz et MYlRea,I963 ; Bouissou, I965; PORZIG, I965; Dickson et al., I965, I967...).

Par hiérarchie sociale, il faut entendre la subordination de certains individus à certains autres, à la suite de combats, de menaces, de soumission passive ou de la combinaison de ces trois éléments.

Pour un observateur, cette hiérarchie se traduit par des luttes, des coups, des attitudes de menace, des préséances lorsqu'il y a compétition ou plus simplement par le fait qu'un animal s'écarte à l'approche d'un autre et lui cède le pas. 
L'ordre social une fois établi conduit à un équilibre et des manifestations relativement inoffensives remplacent les luttes proprement dites. Cependant, même dans ces conditions, l'existence des animaux dominés est affectée dans un certain nombre de domaines ; leur temps d'alimentation en particulier est considérablement réduit par rapport à celui des animaux dominants (Bouissou, I964; PoRzIG, I965; Bosc, Bouissou, SignoRet, I968), et leur production laitière peut également être affectée (Porzig et Wenzel, I969; Danilo, I969).

L'étude expérimentale des signaux intervenant dans la manifestation des relations de dominance-soumission est nécessaire pour prévenir les conséquences défavorables de l'existence d'une hiérarchie, et y remédier dans la pratique de l'élevage.

Nous avons tout d'abord cherché à préciser le rôle des signaux visuels et du contact physique; en effet, l'extériorisation de la hiérarchie existante n'implique pas forcément un contact physique entre les animaux et des signaux agissant à distance interviennent souvent seuls (Schein et Fohrman, I955 ; GuHI, et AtKeson, I959; Bourssou, I965). I1 semblait donc légitime d'attribuer un rôle important à la vue; le contact physique, au contraire, semblait jouer un rôle moindre. Les résultats (Bouissou, I968, non publiés) ont montré que la vue, si elle apporte une information, n'est pas nécessaire à la manifestation des relations hiérarchiques. Par contre, le contact physique bien que n'intervenant pas obligatoirement, doit être possible : l'interposition entre deux animaux d'une séparation leur permettant néanmoins de se voir et de se sentir, perturbe totalement les manifestations de la hiérarchie et efface ses conséquences sur le temps d'alimentation lors d'épreuves de compétition, au contraire de ce qui se passe chez certaines espèces, même plus évoluées, comme les Primates (WARDEN et GaLT, I943).

Il semble donc exister chez les Bovins ce que nous nommerons une "notion de protection " vis-à-vis de l'animal dominant.

Étant donné l'importance pratique de ce phénomène dans la conception des installations destinées aux animaux d'élevage, il nous a paru nécessaire d'étudier expérimentalement cette " notion de protection " en simplifiant progressivement la séparation dont l'efficacité a été démontrée.

\section{MATÉRIEL E'T MÉTHODES}

$$
\text { A. - Les animanx }
$$

Cette étude a porté sur six génisses de race Française Frisonne Pie-Noire, âgées de 3 ans, entretenues ensemble en stabulation libre depuis l'âge de six mois.

\section{B. - Épreuves de compétition}

Nous avons utilisé une méthode de compétition alimentaire par paire (Bourssou, 1970) en nous plaçant dans les conditions de l'alimentation à l'auge.

Un aliment concentré était proposé à un couple de génisses dans l'auge où elles recevaient habituellement leur repas; l'aliment était placé dans un compartiment de $70 \mathrm{~cm}$ de long, de telle sorte que les deux animaux puissent s'alimenter en même temps, mais en étant en contact physique étroit. Le temps d'alimentation de chaque animal était mesuré au cours d'un test de trois minutes.

Quatre situations expérimentales, définies par le type de séparation interposée entre les animaux, ont été comparées à la situation " témoin ", c'est-à-dire sans séparation. 
- Séparation de 2,50 $\mathrm{m}$ de long, constituée de barres horizontales distantes de $20 \mathrm{~cm}$, placée au-dessus et en arrière de l'auge.

- Séparation de même type, de $2 \mathrm{~m}$ de long, placée en arrière de l'auge.

- Séparation de même type, de $50 \mathrm{~cm}$ de long, placée au-dessus de l'auge et limitée à celle-ci.

- Enfin, une seule barre de $50 \mathrm{~cm}$ de long, placée à $20 \mathrm{~cm}$ au-dessus de l'auge.

Ces différents dispositifs sont schématisés par la figure 1 .

Les I 5 paires, constituées à partir des 6 animaux expérimentaux, ont été réparties en 5 groupes de 3, de telle sorte qu'un animal ne soit testé chaque jour qu'avec un seul partenaire. L'ordre dans lequel étaient testés les groupes, donc l'ordre dans lequel un animal rencontrait les autres, était déterminé au hasard.

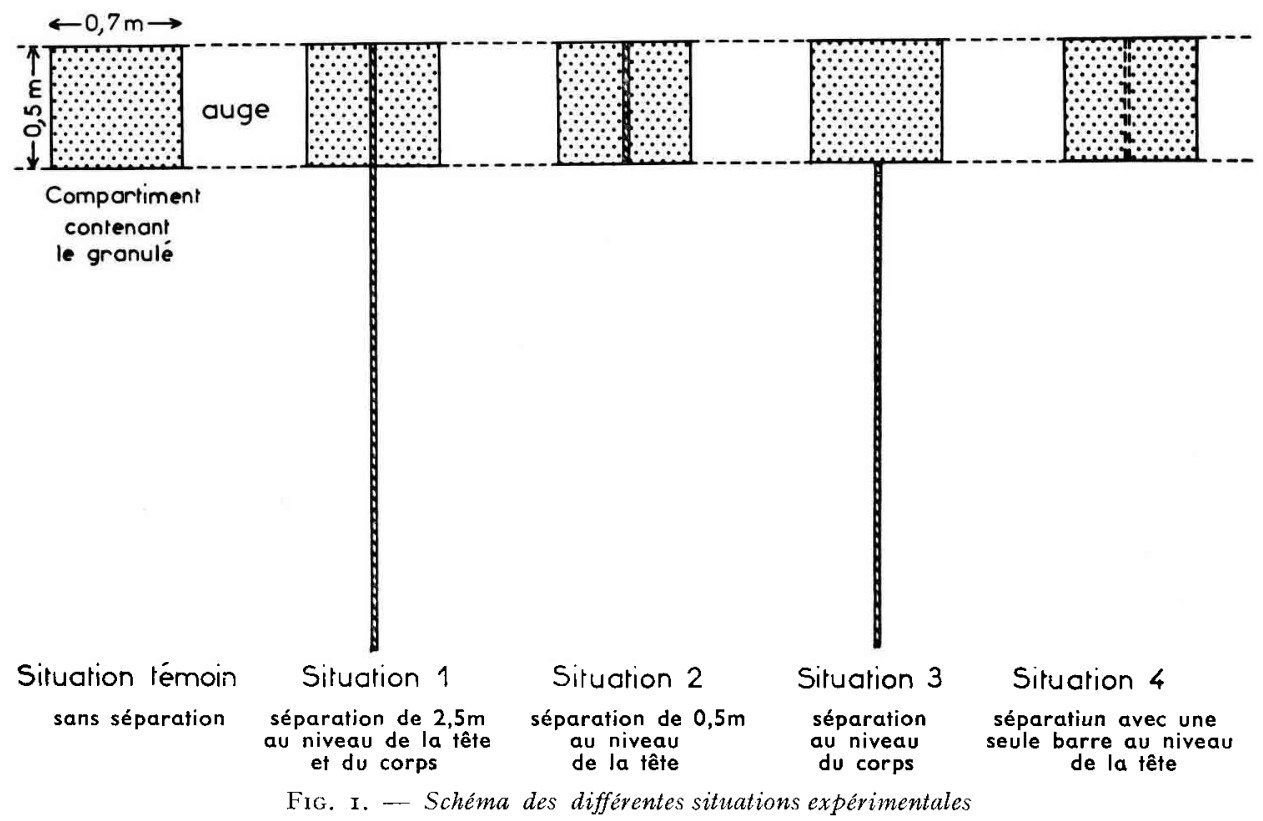

Chaque paire a été testée trois fois dans chacune des situations précédemment décrites. Pour une paire donnée, un test témoin était de nouveau effectué pour chaque série expérimentale. La position de ce test par rapport aux trois tests expérimentaux était déterminée au hasard.

\section{RÉSULTATS}

Lorsque les animaux ne sont pas séparés, l'animal dominant s'alimente pratiquement seul pendant la totalité du test (tab1. I). Dans la majorité des cas, le dominé n'essaie même pas de s'approcher de l'aliment.

L'interposition de la séparation de $2,50 \mathrm{~m}$ réduit notablement la possibilité de contact physique entre animaux, bien que la vache dominante aît toujours la possibilité de la contourner pour chasser la dominée. Dans ces conditions, le temps d'alimentation des animaux dominés augmente considérablement et passe de 7 secondes à 2 mn I3 (tabl. I).

L'efficacité de ce type de séparation étant démontré, nous avons ensuite cherché 
à préciser si la protection devait porter sur l'ensemble du corps ou si certaines zones privilégiées pouvaient être mises en évidence.

Les résultats ont montré que la séparation au niveau du corps seul n'a guère d'effet: la dominée ne mange en moyenne que 4I secondes. Par contre, la séparation au niveau de la tête conduit à une augmentation du temps d'alimentation des animaux dominés de même ordre que la séparation complète. Eñfin, une simple barre au niveau de la tête permet également une sensible amélioration du temps d'alimentation des animaux dominés par rapport à la situation témoin (tabl. I).

Alors que précédemment nous avions envisagé le temps moyen d'alimentation des dominantes et des dominées de chaque paire, nous pouvons calculer le temps moyen d'alimentation de chaque animal opposé à tous les autres, afin d'avoir une estimation de la situation au sein du groupe (fig. 2).

Dans le cas où il n'y a pas de séparation, la vache dominante du groupe (ou animal a) mange en moyenne, au cours de tous les tests, $2 \mathrm{mn} 58$; les temps d'alimentation des autres animaux se répartissent dans un ordre décroissant correspondant à l'ordre hiérarchique jusqu'à celui de l'animal dominé (ou animal $\omega$ ) qui n'est que de 39 secondes. L'écart entre les temps d'alimentation de l'animal dominant et de l'animal dominé est donc de $2 \mathrm{mn}$ rg. Cet écart est moindre pour chacune des quatre autres situations, en particulier pour la séparation de $2,50 \mathrm{~m}$ et la séparation de $50 \mathrm{~cm}$ au niveau de la tête, où il est respectivement de 35 secondes et 40 secondes. Par ailleurs, dans le cas où la séparation est réduite à une barre, la réduction de cet écart est surtout sensible pour les deux derniers animaux de la hiérarchie (fig. 2).

La protection de la tête est donc l'élément essentiel qui permet au dominé un accès à l'aliment malgré la présence du dominant.

\section{TABLEAU I}

Effet des différents types de séparations sur les temps moyens d'alimentation de génisses opposées par paires dans des tests de compétition de $3 \mathrm{mn}$

\begin{tabular}{|c|c|c|c|c|c|}
\hline$i$ & $\begin{array}{c}\text { Situation } \\
\text { témoin } \\
\text { (sans séparation) }\end{array}$ & $\begin{array}{l}\text { Situation } \\
1 \\
\text { (sép. niveau } \\
\text { tête et corps) }\end{array}$ & $\begin{array}{c}\text { Situation } \\
2 \\
\text { (sép. niveau } \\
\text { tête) }\end{array}$ & $\begin{array}{l}\text { Situation } \\
\quad 3 \\
\text { (sép. niveau } \\
\text { corps) }\end{array}$ & $\left(\begin{array}{c}\text { Situation } \\
/ 4 \\
(1 \text { seule barre })\end{array}\right.$ \\
\hline $\begin{array}{l}\text { Moyenne des temps } \\
\text { d'alimentation des } \\
\text { dominantes }\end{array}$ & $2 \mathrm{mn} 57 \mathrm{~s}$ & $2 \mathrm{mn} 5 \mathrm{~s} \mathrm{~s}$ & $2 \mathrm{~mm} 58 \mathrm{~s}$ & $2 \mathrm{mnl} 48 \mathrm{~s}$ & $2 \mathrm{mn} 5 \mathrm{x}$ \\
\hline $\begin{array}{c}\text { Moyenne des temps } \\
\text { d'alimentation des } \\
\text { dominées }\end{array}$ & $7 \mathrm{~s}$ & $2 \mathrm{mn} 13 \mathrm{~s}$ & $2 \mathrm{mn} \ddot{2}$ & $41 \mathrm{~s}$ & I mne's \\
\hline
\end{tabular}




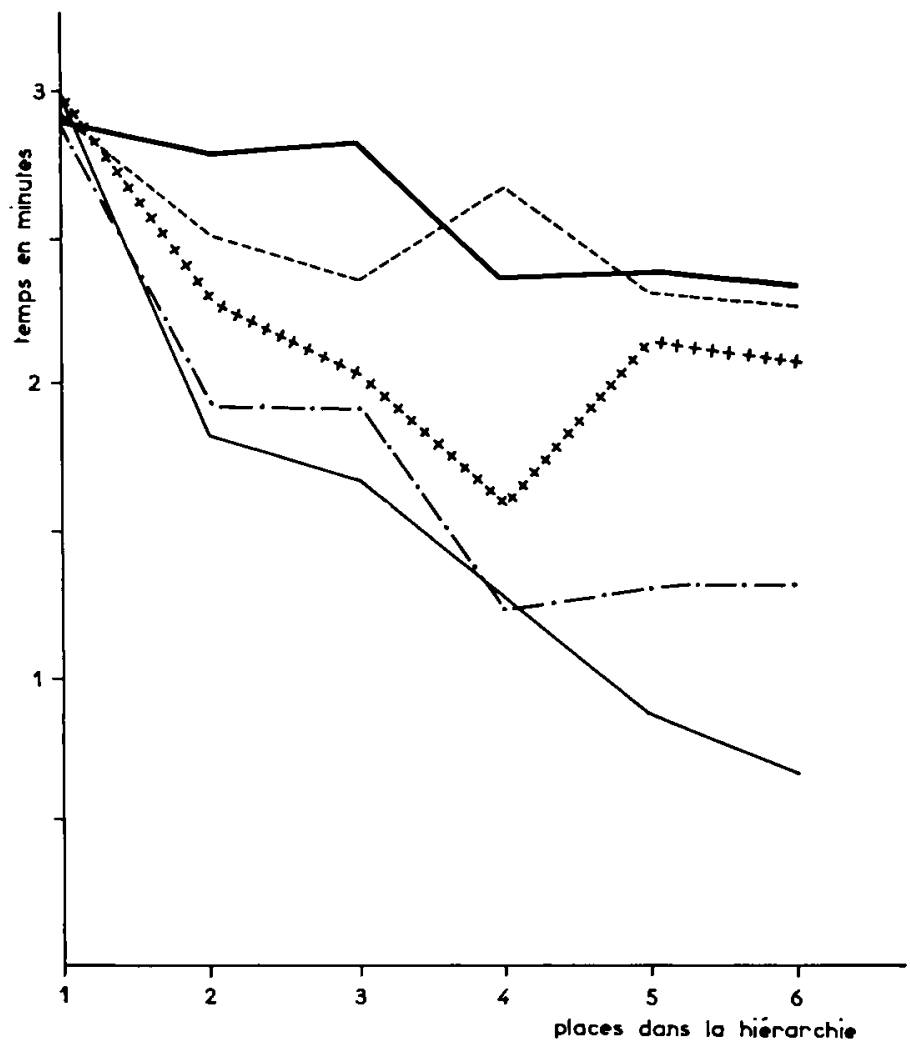

FIG, 2. - Temps moyen d'alimentation de chaque animal opposé à tous les autres, en fonction de son rang hiérarchique.

sans séparation

avec séparation de $\mathbf{2 , 5} \mathrm{m}$ au niveau de la tête et $\mathbf{d u}$ corps

- - - - - - avec séparation de $0,5 \mathrm{~m}$ au niveau de la tête

- $\cdot-\cdot-\ldots \cdot-$ avec séparation de $2 \mathrm{~m}$ en arrière de l'auge

++++++ avec une seule barre au niveau de la tête

\section{CONCLUSION ET DISCUSSION}

L'étude expérimentale du rôle de différents stimuli lors de la manifestation des relations de dominance-soumission, nous a permis de mettre en évidence l'importance de la possibilité du contact physique entre les animaux.

Une protection de l'animal dominé est nécessaire pour minimiser les conséquences défavorables d'une compétition alimentaire avec un animal de rang plus élevé. Nous avons montré que cette protection conservait toute son efficacité lorsqu'elle est limitée à la tête ; il est même possible de la réduire à un niveau " symbolique ".

L'alimentation à l'auge des Bovins entretenus en stabulation libre crée une situation de compétition qui, si elle n'atteint pas l'intensité de celle réalisée dans nos conditions expérimentales est cependant importante. Les résultats précédents peuvent remettre en question l'efficacité des dispositifs actuellement adoptés; les cor- 
nadis en particulier, permettant une libre interaction aux têtes des animaux, n'empêchent vraisemblablement pas le dominant de contrôler une portion plus grande de l'auge.

Ces résultats fournissent des bases à une étude rationnelle des aménagements à proposer pour la distribution des aliments aux Bovins. Il serait intéressant de les mettre à l'essai dans la pratique.

Cette " notion de protection" est probablement importante dans un certain nombre d'autres situations. Ainsi, l'augmentation du temps de repos que nous avons observé dans certaines stabulations à logettes, par rapport à la stabulation libre, est à rapprocher des résultats précédents et peut s'expliquer de manière analogue.

Cette notion de l'importance du contact physique devrait également être prise en considération pour l'élaboration ou l'aménagement de différents types d'installations telles que les étables à logettes ou les salles de traite par exemple.

\title{
Reçu pour publication en juillet 1970.
}

\author{
SUMMARY \\ THE ROLE OF PHYSICAL CONTACT IN THE MANIFESTATION \\ OF HIERARCHICAL RELATIONSHIPS IN CATTLE \\ PRACTICAL CONSEQUENCES
}

The experimental study of dominance-submission signals in domestic cattle previously indicated the important role of the possibility of a physical contact between animals. The total absence of this possibility allows to efface the unfavourable consequences of a hierarchy on the feeding time of the dominated animals to be eliminated, when in competition with a dominant.

This study attemps to determine, under the conditions of trough feeding, which device could improve the feeding time of the dominated animals and which part of the body should be protected.

During competition tests lasting $3 \mathrm{mn}$ and with a through lenght of $70 \mathrm{~cm}$, a trellissed traverse $(2,50 \mathrm{~m}$ long) situated above and behind the through permits the mean feeding time of dominated animals to be increased from $7 \mathrm{~s}$ to $2 \mathrm{mn} \mathrm{I} 3 \mathrm{~s}$. If the separation is reduced to the posterior part, that is at the level of the neck and the body of the animals, the arevage feeding time of the dominated is only ${ }_{4} \mathrm{I}$ s. This mean increases to $2 \mathrm{mn} 2 \mathrm{~s}$ when the separation is limited to the anterior part, that is at the level of the head. Finally, a simple transverse bar situated at $20 \mathrm{~cm}$ above the through also allows a distinct improvement in feeding time of dominated animals ( $\mathrm{I} \mathrm{mn} 24 \mathrm{~s}$ ). Thus, the protection of the head is essential.

\section{RÉFÉRENCES BIBLIOGRAPHIQUES}

Bjeilharz R. G., Mylrea P. J., 1963a. Social position and behavior of dairy heifers in yards. Anim. Behav., 11, 522-527.

Beilharz R. G., Mylrea P. J., I $963 b$. Social position and movement orders of dairy heifers. Anim. Behav., 11, 529-533.

Bosc M., Bourssou M.-F., Signoret J.-P., rg68. Conséquences de la hiérarchie sociale sur le comportement alimentaire des Bovins domestiques. $94^{\mathrm{e}}$ Cong. Nat. Soc. Savantes, Tours.

Bouissou M.-F., I964. Observations sur la hiérarchie sociale chez les bovins domestiques. D. E. S. Faculté des Sciences, Paris (non publié).

Bourssou M.-F., rg65. Observations sur la hiérarchie sociale chez les bovins domestiques. Ann. Biol. anim. Bioch. Biophys., 5, 327-339. 
Bouissou M.-F., 1970. Technique de mise en évidence des relations hiérarchiques dans un groupe de Bovins domestiques. Rev. Comp. Anim., 4, 66-69.

Danilo M., I969. (Observations non publiées.)

Dickson D. P., Barr G. R., Wieckert D. A., I967. Social relationships of dairy cows in a feed lot. Behaviour, $29(2-4)$, $195-203$.

Guhl A. M., Atkeson F. W., 1959. Social organization in a herd of dairy cows. Trans. Kans. Acad. Sci., 62 (I), 80-87.

McPhe C. P., Mcbride G., James J. W., I964. Social behaviour of domestic animals. III. Steers in small yards. Anim. Prod., 6, 9-I5.

Porzig E., I965. Vorläufige Ergebnisse zum Einfluss der Sozialen Rangordnung auf das Verhalten des Haustindes. Karl Marx Univ., Kolloquium, 25-26-3-65.

Porzig E., WENzEL G., r969. Verhalten der Milchkühe nach der Umstellung aus dem Abkalbestall in den Boxenlaufstall. Tierzücht, 23, 535-537.

Schein M. W., Fohrman M. H., 1955. Social dominance relationships in a herd of dairy cattle. British J. Anim. Behav., 3 (2), 45-55.

TulloH N. M., I961. Behaviour of cattle in yards; weighing order and behaviour before entering scales. Anim. Behav, $9(\mathbf{1}-2), 20-24$.

WARDEN C. J., GALT W., 1943. A study of cooperation, dominance, grooming and other social factors in monkeys. J. Genet. Psychol., 63, $213-233$. 